\title{
Risikoabschätzung - Prognostische Informationen für die Aufklärung in der klinischen Praxis
}

\author{
Martin et al. werteten die Daten von 12.271 Patienten hinsicht- \\ lich der Inzidenz und der Risikofaktoren für postoperative Kom- \\ plikationen nach Kniearthroskopie aus. Dabei zeigte sich, dass \\ die Komplikationsrate maßgeblich durch das Patientenalter, \\ den Body-Mass-Index und vorangegeangene OPs beeinflusst \\ wird. \\ Martin CT et al. Risk factors for thirty-day morbidity and mortality following knee arth- \\ roscopy: a review of 12,271 patients from the national surgical quality improvement \\ program database. J Bone Joint Surg Am 2013; 95: e98 (1-10)
}

\section{Einleitung \\ $\nabla$}

Arthroskopische Eingriffe am Kniegelenk gehören zu den am häufigsten durchgeführten orthopädischen Operationen. Im klinischen Alltag werden diese Eingriffe als „komplikationsarm“ angesehen. Genaue Angaben bezüglich der Inzidenz von Komplikationen nach Kniearthroskopie und möglicher Risikofaktoren für das Auftreten sind bisher nur in geringen Maße vorhanden.

\section{Methodik}

Im Rahmen der vorliegenden Studie wurden prospektiv erhobene epidemiologische und medizinische Daten von 12271 Patienten über das American College of Surgeons Quality Improvement Program ausgewertet. Die Patienten erhielten zwischen Januar 2005 und Dezember 2010 eine elektive Kniegelenksarthroskopie in einem an der Datenerhebung beteiligten Krankenhaus in den USA. Die Datenanalyse erfolgte retrospektiv unter Verwendung der Procedurencodes.

Es erfolgte eine Unterteilung der Eingriffe in 2 Schweregrade. Als komplexere Eingriffe wurden dabei Arthroskopien mit gleichzeitiger Durchführung eines vorderen oder hinteren Kreuzbandersatzes,
Meniskustransplantation oder Aufbringen eines osteochondralen Allo- oder Autografts gewertet. Komorbiditäten und binnen 30 Tagen postoperativ aufgetretene Komplikationen (unterschieden in 2 Schweregrade und Mortalität) wurden ebenfalls erfasst. Durch uni- und multivariante Analysen wurden potentielle Risikofaktoren statistisch ausgewertet.

\section{Ergebnisse \\ $\nabla$}

Es zeigten sich bei insgesamt 199 Patienten $(1,6 \%) 240$ postoperative Komplikationen. Schwere, sprich das Organsystem bedrohende, Komplikationen, einschließlich Knieinfektion (0,12\%) und Sepsis $(0,06 \%)$ traten bei $0,76 \%$ der Patienten auf. $0,62 \%$ erhielten eine Revisionsoperation. $0,86 \%$ erlitten weniger schwere Komplikationen, einschließlich tiefer Venenthrombosen oder Thrombophlebitis (0,46\%) und oberflächlicher Wundinfektionen $(0,27 \%)$. Ein Patient verstarb nach Durchführung des Eingriffes.

Das Auftreten von Komplikationen war von der Komplexität des Eingriffes unabhängig. In der univarianten Analyse konnte gezeigt werden, dass das Outcome aller Komplikationen deutlich schlechter war für Patienten dunkler Hautfarbe $(\mathrm{p}=0,013)$, bei präoperativer Dyspnoe
( $p<0,0001)$, Diabetes mellitus $(p=0,016)$ sowie nach vorangegangenen Operationen binnen der letzten 30 Tage $(\mathrm{p}=0,048)$. Als unabhängige Risikofaktoren für alle Komplikationen konnten bei Patienten dunkler Hautfarbe in der multivarianten Analyse (odds ratio 1,81, 95\% Konfidenzintervall 1,13 bis 2,89) vorangegangene Operationen binnen der letzten 30 Tage (OR 6,33, $95 \%$ KI 1,45 bis 27,66), Operationszeit länger als 1,5 Stunden (OR 1,84, $95 \% \mathrm{KI} 1,21$ bis 2,78) sowie Alter zwischen 40 und 65 Jahren (OR 1,46, 95\% KI 1,01 bis 2,11 ) benannt werden. Für das Outcome schwerer Komplikationen waren in der univarianten Analyse insbesondere das Patientenalter $(p=0,027)$ und ein BodyMass-Index über $35(\mathrm{p}=0,025)$ bedeutend. In der multivarianten Analyse wurden eine präoperative Dyspnoe (OR 3,13, 95\% KI 1,53 bis 6,4) und Auffälligkeiten in der Blutungsanamnese (OR 3,79, 95\% KI 1,28-11,2) hervorgehoben.

\section{Kommentar \\ $\nabla$}

Insgesamt betrachtet ist die Komplikationsrate bei arthroskopischen Eingriffen am Kniegelenk gering, keinesfalls jedoch unbedeutend. Interessanterweise spielte die Komplexität des operativen Eingriffes für das Auftreten einer Komplikation insgesamt keine Rolle. Aus den präsentierten Daten der Untersuchung lassen sich prognostische Informationen ableiten, die bei der Aufklärung des Patienten für den operativen Eingriff berücksichtigt werden sollten. Elektive Eingriffe binnen 30 Tagen nach Durchführung einer anderen Operation sind nicht empfehlenswert. Außerdem ist eine möglichst kurze Operationszeit anzustreben. Patienten mit präoperativer Dyspnoe, mit bekanntem Diabetes mellitus sowie mit einem BMI über 35 sollten nur nach entsprechender RisikoNutzen-Abwägung operiert und entsprechend überwacht werden. Die ambulante Durchführung von arthroskopischen Operationen am Kniegelenk ist bei diesen $\mathrm{Pa}$ tienten entsprechend kritisch zu sehen.

Dr. med. Kerstin Radtke

Orthopädische Klinik der Medizinischen

Hochschule Hannover im Annastift

kerstin.radtke@ddh-gruppe.de 